\title{
Effect of static stretching with different rest intervals on muscle stiffness.
}

\section{AUTHOR(S):}

Nojiri, Shusuke; Ikezoe, Tome; Nakao, Sayaka; Umehara, Jun; Motomura, Yoshiki; Yagi, Masahide; Hirono, Tetsuya; Ichihashi, Noriaki

\section{CITATION:}

Nojiri, Shusuke ...[et al]. Effect of static stretching with different rest intervals on muscle stiffness.. Journal of biomechanics 2019, 90: 128132

\section{ISSUE DATE:}

2019-06-11

URL:

http://hdl.handle.net/2433/243829

\section{RIGHT:}

(c) 2019. This manuscript version is made available under the CC-BY-NC-ND 4.0 license

http://creativecommons.org/licenses/by-nc-nd/4.0/; The full-text file will be made open to the public on 11 June 2020 in accordance with publisher's 'Terms and Conditions for Self-Archiving'.; This is not the published version. Please cite only the published version.; この論文は出版社版でありません。引用の際には出版社版をご確認ご利用ください。 


\section{Effect of static stretching with different rest intervals on muscle stiffness}

Corresponding author:

$9 \quad$ Shusuke Nojiri

Human Health Sciences, Graduate School of Medicine, Kyoto University

53 Kawahara-cho, Shogoin, Sakyo-ku, Kyoto 606-8507, Japan.

E-mail: nojiri.shusuke.35v@st.kyoto-u.ac.jp

Office phone: +81-75-751-3951

Office fax: +81-75-751-3951 


\section{ABSTRACT (244/250 words)}

The aim of the study was to investigate the effect of static stretching (SS) with different rest intervals on muscle

stiffness. Fifteen healthy males participated in the study. Four bouts of thirty-second SS for the gastrocnemii were

performed at the maximal dorsiflexion using dynamometer with two different rest intervals between stretches,

namely $0 \mathrm{~s}$ (R0) and $30 \mathrm{~s}$ (R30). Each participant underwent both stretching protocols at least 48 hours apart in a

random order. Between each bout of SS, the ankle was moved to $20^{\circ}$-plantar-flexion in $3 \mathrm{~s}$, held for each rest

gastrocnemius was measured before (PRE) and immediately after (POST) four bouts of SS to assess muscle

stiffness of the medial gastrocnemius. Two-way repeated measures analysis of variance (protocol× time) indicated

\pm 5.2 \%); R30, PRE: $11.0 \pm 2.8 \mathrm{kPa}$, POST: $10.2 \pm 2.1 \mathrm{kPa}$, amount of change: $0.8 \pm 1.3 \mathrm{kPa}(6.0 \pm 10.4 \%)]$. 


\section{Introduction}

Static stretching (SS) is an effective intervention to decrease the stiffness of a muscle or muscle-tendon unit (MTU)

and to improve the joint range of motion (ROM) (Kay et al., 2015; Konrad et al., 2017; Nakamura et al., 2011).

Since increased stiffness is considered a risk factor of musculoskeletal injuries, SS is often performed prior to performance to prevent injuries (Herbert et al., 2011; McHugh and Cosgrave, 2010).Therefore, investigating the acute effects of SS is important. With respect to an appropriate SS time to decrease MTU stiffness, a previous study demonstrated that at least 2 min of SS was required to decrease the passive torque of gastrocnemii (Nakamura et al., 2013). In clinical situations, SS is typically divided into multiple repetitions as opposed to being performed continuously for a few minutes (Baechle, 1994), presumably to its ease to perform for therapists and high compliance for patients. When SS is divided into multiple repetitions, it is necessary to consider the total stretching time (i.e., SS time per repetition and number of repetitions) and rest interval time between repetitions. With respect to the total time, a previous study indicated that SS for a constant total time with different time per repetition and number of repetitions (i.e., $60 \mathrm{~s} \times 2$ times, $30 \mathrm{~s} \times 4$ times, and $10 \mathrm{~s} \times 12$ times) causes similar effects corresponding to decreases in stiffness of the gastrocnemii muscles (Nakamura et al., 2017). Conversely, few studies investigated the influence of rest interval time between repetitions. Freitas et al. (2015) compared the improvement in ROM between SS with and without rest interval, and concluded that SS without rest interval was more effective in terms of improving ROM. However, the results of the aforementioned study indicated that the effects of ROM improvement were different based on number of repetitions because the number of repetitions (i.e., total time) was 
not consistent among participants. Therefore, it is important to investigate the influence of rest interval time between repetitions while holding the total time of SS as a constant.

Increased stiffness of the muscle or MTU has been reported to increase the risk of musculoskeletal injuries (Watsford et al., 2010). It is noted that ROM is insufficient to assess passive mechanical properties because it is also affected by pain and stretch tolerance (Weppler and Magnusson, 2010). A few studies (Halbertsma and Göeken, 1994; Magnusson et al., 1996; McNair et al., 2001) have indicated that the increase in ROM induced by SS might be due only to a change in stretch tolerance without change in passive mechanical properties (so-called 'Sensory Theory’ reviewed by Weppler and Magnusson, 2010). Since SS is performed to change not only ROM but also the passive mechanical properties of a muscle or MTU, the effects of SS on passive mechanical properties should be distinguished from stretch tolerance. Hence, passive joint stiffness (which is determined as a slope of torque-angle relationship (Magnusson et al., 1996)) and muscle stiffness (which is represented by shear elastic modulus) are used as indices of stretching effects to assess passive mechanical properties. Passive joint stiffness reflects several factors in addition to muscle stiffness such as the stiffness of joint capsules and ligaments (Maïsetti et al., 2012). Shear elastic modulus measured via ultrasound shear wave elastography (SWE) non-invasively makes it possible to quantitatively assess the muscle stiffness of an individual muscle. Therefore, shear elastic modulus is often used as an index of stretching effect for several skeletal muscles (Ichihashi et al., 2016; Kusano et al., 2017; Xu et al., 2018). Thus, we focused on the stiffness of individual muscles among the passive mechanical properties. 
71 in muscle stiffness.

stiffness of the medial gastrocnemius. Our hypothesis is that SS with shorter rest intervals leads to a greater decrease

\section{Methods}

\subsection{Participants}

Fifteen healthy men (height, $171.4 \pm 6.2 \mathrm{~cm}$; mass, $66.7 \pm 9.2 \mathrm{~kg}$; age, $24.3 \pm 3.0$ years) participated in the study.

The sample size required for a two-way repeated measures analysis of variance (ANOVA) [effect size $=0.40$ (large),

$\alpha$ error $=0.05$, power $=0.80]$ was calculated in advance via $G^{*}$ power software (version 3.1.; Heinrich Heine University, Düsseldorf, Germany), and the calculated sample size corresponded to 14 . The effect size was determined based on a previous study, which showed the effects of SS on muscle stiffness using two-way analysis of variance (Akagi and Takahashi, 2014). All participants received an explanation about the study and provided written informed consent. The study was approved by the ethics committee of Kyoto University Graduate School and the Faculty of Medicine (R0233-3).

\subsection{Experimental protocol}

The experimental design was a cross-over design wherein each participant underwent both stretching protocols at least 48 hours apart in a random order. The participants were instructed to maintain their regular physical activities, avoiding unusual exercise between the two sessions. Thirty-second SS for the triceps surae, especially the gastrocnemii in the right leg was repeated for four bouts in the following two protocols: 0-s rest interval (R0) and 
90

91

30-s rest interval (R30) between each bout of SS. The shear elastic modulus of the medial gastrocnemius (MG) was measured before (PRE) and immediately after (POST) four bouts of SS.

The participants lay prone on a dynamometer (BIODEX System 4, Biodex, USA) with the hip in a neutral position (without any flexion/extension, adduction/abduction, or internal/external rotation) and the knee fully extended, and the foot was attached securely to the footplate of the dynamometer (Fig.1a, b). To define the final angle, the ankle was passively dorsiflexed at $5 \%$ s starting from $30^{\circ}$-plantar-flexion to the maximal dorsiflexion angle (Fig. 1b) that the participants achieved without discomfort or pain (Nakamura et al., 2014). The participants themselves stopped the dynamometer via a remote button. The maximal dorsiflexion angle was defined as the final angle in the study and used for all four bouts of SS.

Surface electromyography (EMG) (TeleMyo2400, Noraxon USA, Scottsdale, AZ, USA) on the lateral gastrocnemius muscle belly was used to ensure that the muscle was inactive during SS and measurements of shear elastic modulus. EMG data was calculated using full-wave rectification and the root-mean-square, with a window interval of $50 \mathrm{~ms}$. Then, the EMG activities during SWE measurements and SS were represented as a percentage of the maximal EMG values during maximal voluntary contraction, which was performed after all other protocols.

\subsection{Measurement of shear elastic modulus}

The shear elastic modulus of MG was measured to assess muscle stiffness via an ultrasound SWE (Aixplorer, SuperSonic Imagine, France) with a linear probe (4-15 MHz, SuperLinear 15-4, France) in Musculoskeletal (MSK) 
preset. The measurements were performed in a neutral ankle position $\left(0^{\circ}\right.$ plantar-flexion). The measurement site

was defined at a level corresponding to proximal $30 \%$ of the lower leg length from the popliteal crease to the lateral

malleolus in accordance with previous studies (Akagi and Takahashi, 2013; Nakamura et al., 2014). After

identifying MG on the ultrasound B-mode image, the measurement site was marked on the skin with a pen to ensure

that PRE and POST measurements are performed on the same site. The orientation of the probe was adjusted to the

belly bulge of MG, and the image was then obtained. Two images were obtained at each PRE and POST time point.

The location of the ROI was kept constant for each participant. The probe was repositioned between capturing two 
95\% confidence interval (CI) was calculated from the shear elastic modulus of the two measurements at each time

point. ICC I $_{1,2}$ values were 0.985 (95\% CI: 0.956-0.995) and 0.970 (95\% CI: 0.912-0.990) at PRE and POST,

respectively; therefore, good reliability was observed (Portney and Watkins, 2000).

\subsection{Static stretching}

The participants received SS in a prone position with the hip in a neutral position and the knee fully extended. The carefully monitored these movements with a stopwatch to ensure that the ankle was moved in $3 \mathrm{~s}$ at each repetition.

\subsection{Statistical analysis}

140 Statistical analysis was performed via SPSS Statistics (version 22; IBM, Armonk, NY, USA). After confirming the normal distribution of each variable via the Shapiro-Wilk test, the following analyses were performed. The final 
factors [protocol $(\mathrm{R} 0, \mathrm{R} 30) \times$ time $(\mathrm{PRE}, \mathrm{POST})]$ was performed. When a significant interaction effect was obtained,

\section{Results}

The EMG activities of lateral gastrocnemius were $<5 \%$ of maximal voluntary contraction in all participants, indicating that the muscle was almost inactive during SS and SWE measurements. The final angle corresponded to between protocols $(\mathrm{p}=0.289)$, indicating that SS was performed at almost the same angle in the two protocols. indicated a significant interaction effect $(\mathrm{p}=0.023, \mathrm{~F}=6.56$, effect size $=0.319)$. Post-hoc paired t-tests comparing 
162

163

164

165

0.023 and 0.031 , respectively).

\section{Discussion}

In this study we investigated the effect of four bouts of 30-s SS with different rest intervals ( $0 \mathrm{~s}, 30 \mathrm{~s})$ on muscle stiffness of MG, and the results indicated that the decrease in muscle stiffness observed after SS with 0-s rest interval exceeded the decrease in stiffness after SS with 30-s of rest between stretching intervals. To the best of our knowledge, this is the first study that investigated the effect of rest interval duration between SS repetitions on muscle stiffness given a constant total stretching time. In addition, the muscle stiffness of MG decreased after both stretching protocols. This result is consistent with previous studies (Akagi and Takahashi, 2013; Kay et al., 2015). The reason as to why the decrease in muscle stiffness was smaller in R30 when compared to that in R0 is potentially related to the degree of recovery in muscle stiffness during rest interval between each bout of SS. Stress relaxation is reported as a phenomena caused by SS, which corresponds to a gradual decline in the passive force on the muscle during stretching at a constant angle (Taylor et al., 1990). In a manner similar to the gradual declines in passive force during SS, it is known that the muscle force also gradually recovers after the muscle is released from stretching (Duong et al., 2001). Duong et al., (2001) reported that the decrease in force due to 20-min SS recovered 2 min after SS by approximately 43\%. Another study reported that the decrease in MTU stiffness due to 5 bouts of 1-min SS returned to the baseline within 10 min after SS (Mizuno et al., 2013). In this study, SS with longer rest interval, such as that in R30, resulted in a smaller effect on the decrease in muscle stiffness owing to a certain 
180 duration between stretching repetitions to support our findings.

amount of recovery between SS repetitions, although this recovery did not cancel the SS effect of each repetition.

There are a few limitations in the study. First, the study only investigated the acute effect of SS, and thus the prolonged or long-term effect is unclear. Second, all the participants were healthy young men. Future studies should investigate the influence of rest interval time in SS for different populations and/or in long-term intervention.

Third, the shear elastic modulus was measured on one specific point of MG; therefore, these findings may not necessarily apply to the whole MG. Moreover, we focused only on muscle stiffness and not tendon stiffness. Finally, the effects on ROM or passive torque, that is, MTU stiffness remain unclear. This is because it was not possible to measure them immediately after SS owing to the time spent resetting the experimental setup.

In conclusion, the study investigated the effect of four bouts of 30-s SS with different rest intervals, namely $0 \mathrm{~s}$ and $30 \mathrm{~s}$, on muscle stiffness of the medial gastrocnemius. The results indicated that SS with 0-s rest interval decreased muscle stiffness to a greater extent although SS with 30-s rest interval also decreased muscle stiffness. The results indicated that SS with a shorter rest interval may be more effective in decreasing muscle stiffness. Clinically, SS with shorter rest intervals could be recommended to decrease muscle stiffness from the viewpoint of injury prevention. Future studies are needed to investigate in greater detail the effect of rest interval 


\section{Acknowledgements}

197

We would like to thank Ms.Ibuki and Editage (www.editage.jp) for English language editing. We would like to thank Mr.Pataky for advices on statistical analysis.

\section{Conflict of interest statement}

The authors declare that they have no conflict of interest.

\section{References}

Akagi, R., Takahashi, H., 2014. Effect of a 5-week static stretching program on hardness of the gastrocnemius muscle. Scand. J. Med. Sci. Sports 24, 950-7. https://doi.org/10.1111/sms.12111

Akagi, R., Takahashi, H., 2013. Acute effect of static stretching on hardness of the gastrocnemius muscle. Med. Sci. Sports Exerc. 45, 1348-1354. https://doi.org/10.1249/MSS.0b013e3182850e17

Baechle, T.R., 1994. Essentials of Strength Training and Conditioning. Human Kinetics, Champaign, IL.

Duong, B., Low, M., Moseley, A.M., Lee, R.Y., Herbert, R.D., 2001. Time course of stress relaxation and recovery in human ankles. Clin. Biomech. (Bristol, Avon) 16, 601-607. https://doi.org/10.1016/S0268-0033(01)00043-2

Freitas, S.R., Vaz, J.R., Bruno, P.M., Valamatos, M.J., Andrade, R.J., Mil-Homens, P., 2015. Are rest intervals between stretching repetitions effective to acutely increase range of motion? Int. J. Sports Physiol. Perform. 10, 191-197. https://doi.org/10.1123/ijspp.2014-0192 
mechanical properties of in vivo muscle tissue assessed by supersonic shear imaging. Ultrasound Med. Biol. 36,

789-801. https://doi.org/10.1016/j.ultrasmedbio.2010.02.013

Halbertsma, J.P., Göeken, L.N., 1994. Stretching exercises: effect on passive extensibility and stiffness in short

Herbert, R.D., de Noronha, M., Kamper, S.J., 2011. Stretching to prevent or reduce muscle soreness after exercise.

Hirata, K., Kanehisa, H., Miyamoto-Mikami, E., Miyamoto, N., 2015. Evidence for intermuscle difference in slack

Ichihashi, N., Umegaki, H., Ikezoe, T., Nakamura, M., Nishishita, S., Fujita, K., Umehara, J., Nakao, S., Ibuki, S., 2016. The effects of a 4-week static stretching programme on the individual muscles comprising the hamstrings. J.

Kay, A.D., Husbands-Beasley, J., Blazevich, A.J., 2015. Effects of Contract-Relax, Static Stretching, and Isometric

Konrad, A., Budini, F., Tilp, M., 2017. Acute effects of constant torque and constant angle stretching on the muscle and tendon tissue properties. Eur. J. Appl. Physiol. 117, 1649-1656. https://doi.org/10.1007/s00421-017-3654-5

Koo, T.K., Guo, J.-Y., Cohen, J.H., Parker, K.J., 2013. Relationship between shear elastic modulus and passive muscle force: an ex-vivo study. J. Biomech. 46, 2053-2059. https://doi.org/10.1016/j.jbiomech.2013.05.016 
extension and internal rotation stretching of the shoulder on infraspinatus muscle hardness. J. shoulder Elb. Surg.

Magnusson, S.P., Simonsen, E.B., Aagaard, P., Dyhre-Poulsen, P., McHugh, M.P., Kjaer, M., 1996. Mechanical and physical responses to stretching with and without preisometric contraction in human skeletal muscle. Arch. Phys. Med. Rehabil. 77, 373-8. https://doi.org/10.1016/S0003-9993(96)90087-8

Magnusson, S.P., Simonsen, E.B., Aagaard, P., Kjaer, M., 1996. Biomechanical responses to repeated stretches in

Maïsetti, O., Hug, F., Bouillard, K., Nordez, A., 2012. Characterization of passive elastic properties of the human

McHugh, M.P., Cosgrave, C.H., 2010. To stretch or not to stretch: the role of stretching in injury prevention and performance. Scand. J. Med. Sci. Sports 20, 169-81. https://doi.org/10.1111/j.1600-0838.2009.01058.x

Mizuno, T., Matsumoto, M., Umemura, Y., 2013. Decrements in stiffness are restored within 10 min. Int. J. Sports

McNair, P.J., Dombroski, E.W., Hewson, D.J., Stanley, S.N., 2001. Stretching at the ankle joint: viscoelastic responses to holds and continuous passive motion. Med. Sci. Sports Exerc. 33, 354-8. https://doi.org/10.1097/00005768200103000-00003 
wave elastography study. Ultrasound Med. Biol. 40, 1991-1997.

https://doi.org/10.1016/j.ultrasmedbio.2014.03.024

Nakamura, M., Ikezoe, T., Nishishita, S., Umehara, J., Ichihashi, N., 2017. Acute effect of static stretching on passive and active properties of the gastrocnemius muscle-tendon unit: an investigation based on different repetition https://doi.org/10.1177/036354659001800314

Portney, L., Watkins, M., 2000. Foundations of clinical research: application to practice, 2nd ed. ed. Prentice Hall Health, New Jersey.

Taylor, D.C., Dalton, J.D., Seaber, A. V., Garrett, W.E., 1990. Viscoelastic properties of muscle-tendon units. The biomechanical effects of stretching. Am. J. Sports Med. 18, 300-309.

Nakamura, M., Ikezoe, T., Takeno, Y., Ichihashi, N., 2013. Time course of changes in passive properties of the gastrocnemius muscle-tendon unit during 5 min of static stretching. Man. Ther. 18, 211-215. https://doi.org/10.1016/j.math.2012.09.010

Nakamura, M., Ikezoe, T., Takeno, Y., Ichihashi, N., 2011. Acute and prolonged effect of static stretching on the passive stiffness of the human gastrocnemius muscle tendon unit in vivo. J. Orthop. Res. 29, 1759-63. https://doi.org/10.1002/jor.21445

Watsford, M.L., Murphy, A.J., McLachlan, K.A., Bryant, A.L., Cameron, M.L., Crossley, K.M., Makdissi, M., 2010. A prospective study of the relationship between lower body stiffness and hamstring injury in professional Australian 
273 Weppler, C.H., Magnusson, S.P., 2010. Increasing muscle extensibility: a matter of increasing length or modifying sensation? Phys. Ther. 90, 438-449. https://doi.org/10.2522/ptj.20090012 
Table 1. Shear elastic modulus values of the medial gastrocnemius in each protocol

\begin{tabular}{|c|c|c|c|c|c|c|}
\hline & \multicolumn{4}{|c|}{ Shear elastic modulus (kPa) } & \multirow{2}{*}{$\begin{array}{l}\text { Percentage } \\
\text { change in shear } \\
\text { elastic modulus } \\
\text { (\%) }\end{array}$} & \multirow[b]{2}{*}{ Interaction effect } \\
\hline & PRE & POST & $\begin{array}{c}\text { p-value of } \\
\text { post-hoc test }\end{array}$ & $\begin{array}{c}\text { Change in } \\
\text { shear elastic } \\
\text { modulus }\end{array}$ & & \\
\hline R0 & $11.5 \pm 3.3$ & $10.0 \pm 2.6 * *$ & $\mathrm{p}<0.001$ & $1.6 \pm 0.9 \dagger$ & $13.0 \pm 5.2 \dagger$ & $\mathrm{p}=0.023, \mathrm{~F}=6.559$ \\
\hline R30 & $11.0 \pm 2.8$ & $10.2 \pm 2.1 *$ & $p=0.029$ & $0.8 \pm 1.3$ & $6.0 \pm 10.4$ & effect size $=0.319$ \\
\hline
\end{tabular}

Values are expressed as mean \pm standard deviation

PRE: before SS, POST: immediately after SS

R0: 0-s rest interval, R30: 30-s rest interval

Change in shear elastic modulus $=$ PRE-value - POST-value

Percentage change in shear elastic modulus $=($ change in shear elastic modulus/PRE-value $) \times 100$

* significant difference between time points $(\mathrm{p}<0.05)$

** significant difference between time points $(\mathrm{p}<0.01)$

$\dagger$ significant difference between protocols $(\mathrm{p}<0.05)$ 


\section{Figure captions}

Fig.1 Experimental setup

(a) Participant is in a prone position with the hip in neutral position and the knee fully extended. In determining the

final angle, the participants pressed the remote button to stop the dynamometer.

(b) The foot on the right is attached securely to the footplate of the dynamometer. The axis of the ankle joint corresponds to the axis of the dynamometer rotation.

Fig.2 Typical example of shear wave elastography image

The region of interest (ROI) was set near the center of the muscle belly bulge of the medial gastrocnemius. A 10 speed in the circle.

Fig.3 Experimental protocol of stretching

294 Maximal dorsiflexion angle was defined prior to the stretching.

295 Static stretching for $30 \mathrm{~s}$ at the final angle was repeated for four bouts.

PF: plantar-flexion 

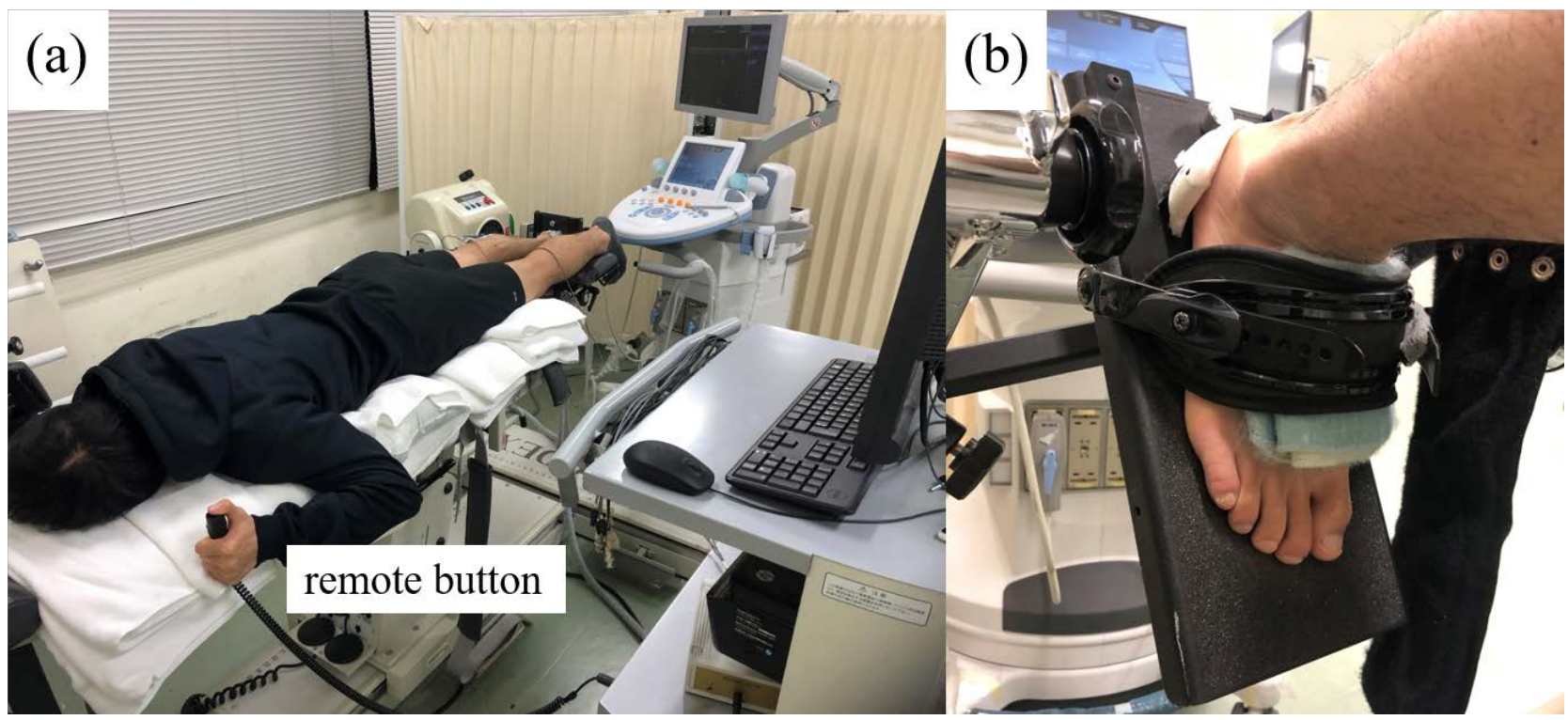

Fig.2

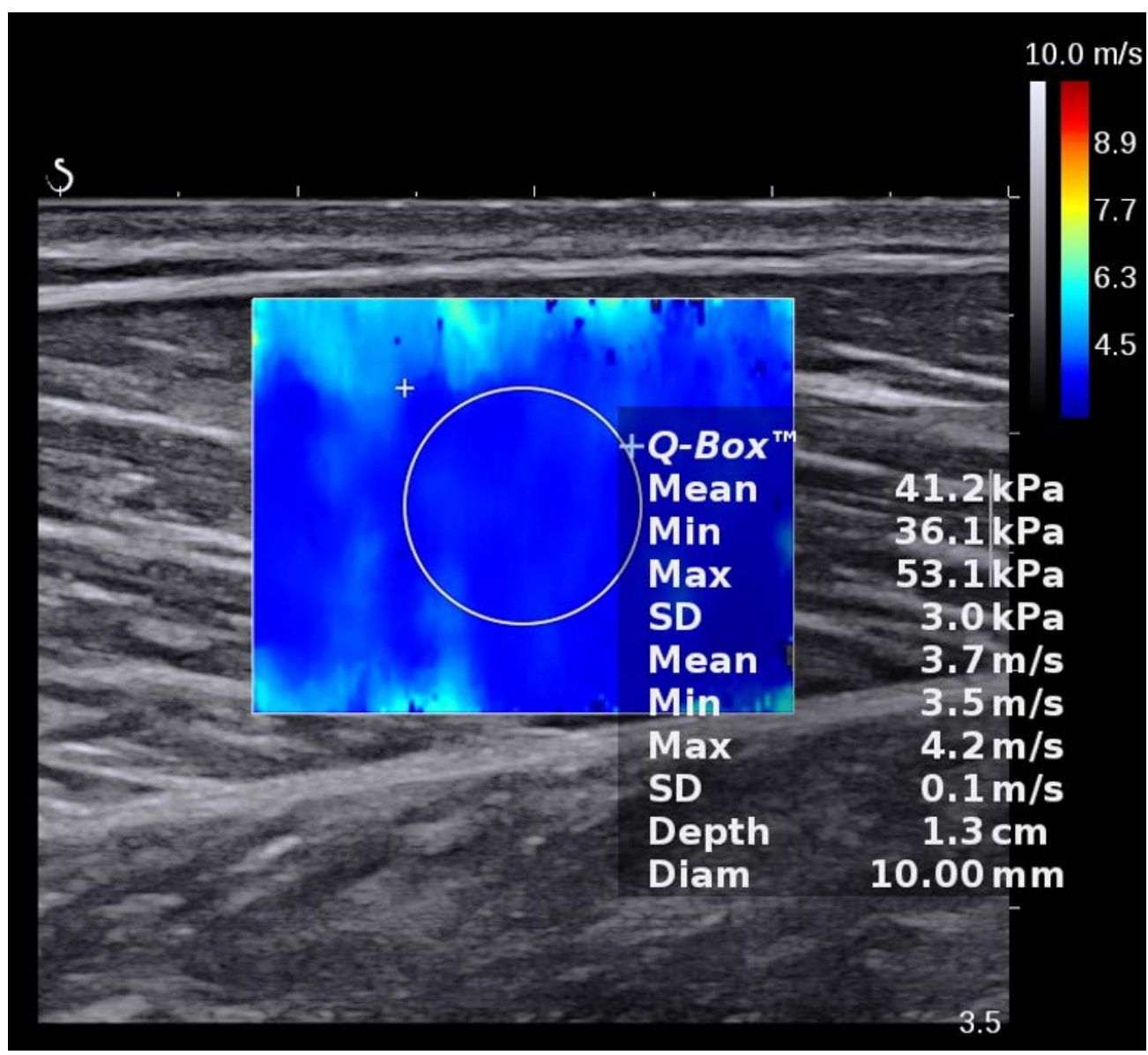




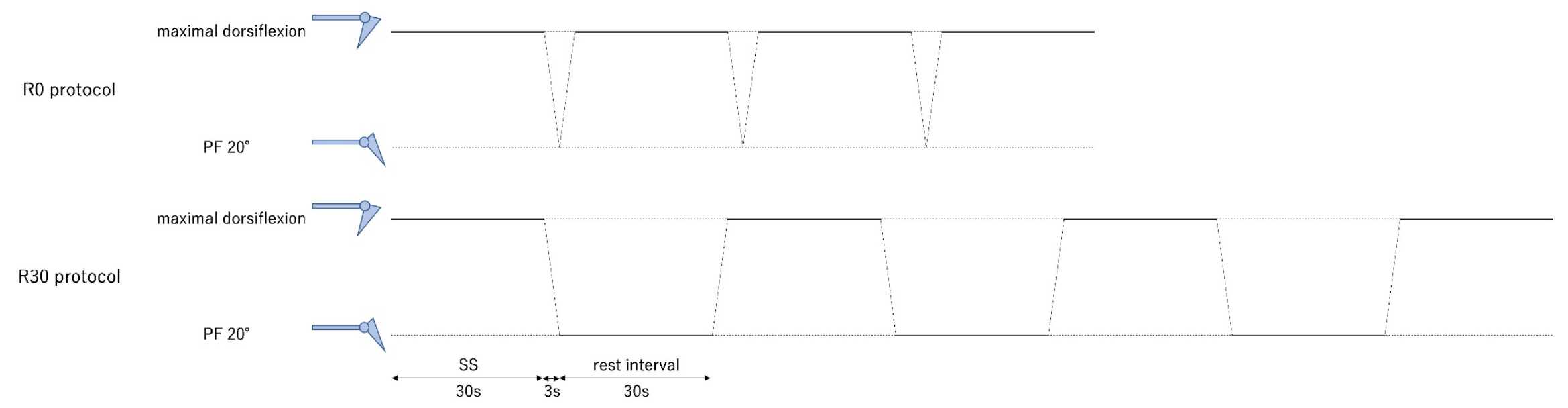

